\title{
An Automated Map Process Based Improved Fuzzy C-Means Algorithm for Pathological Detection in MR Image
}

\author{
S. Vigneshwaran, Vishnuvarthanan Govindaraj, N. Anitha, M. Pallikonda Rajasekaran, \\ Yu-dong zhang, T. Arunprasath
}

\begin{abstract}
Automated brain MR slices segmentation process is difficult, and further difficult is the process of detecting the tumor and tissue regions, with a constraint of delivering higher segmentation accuracy within reduced processing time. Automated algorithms were developed with an onus of reducing the intricacies involved during the manual inspection of the pathologies (radiologist/operator involvement). The shortages of an automated process are overthrown with the development of a novel combination of soft computing algorithms, and it employs automated map and clustering approaches. Self-Organizing map (SOM) and Improved Fuzzy C-Means clustering (IFCM) are the automated map and clustering approaches that are used to precisely provide the MRI slice analysis. The authors have utilized the quality metrics, such as Dice overlap Index (DOI), Jaccard index, Peak Signal to Nosie Ratio (PSNR) and Mean Squared Error (MSE) for verifying the performance of the SOM based IFCM, and the recommended algorithm tenders the corresponding values of the above as $84.83 \%, 91.69 \%, 0.0824$ and 49.25dB. The novel SOM- IFCM algorithm delivers better demarcation outcomes when compared with other soft computing approaches. The exemplified outcomes of the proposed SOMIFCM algorithm provides superior segmentation quality of MR brain slices and offers versatile usage to the radiologists.
\end{abstract}

Keywords : Improved fuzzy c-means clustering, self-organizing map (som), MR brain image analysis, pathological detection, tumor identification.

\section{INTRODUCTION}

$\mathrm{I}_{\mathrm{n}}$ n recent medical image analysis, MR brain slices are mostly supportive for identifying tumor and tissue regions, and at some instances, one cannot easily understand the pathological structures within it (cannot vision the pathological portion due to humor operability). Successively, these lacks can be undone using the soft computing

Revised Manuscript Received on December 16, 2019.

* Correspondence Author

Vigneshwaran*, Department of ECE, Kalasalingam Academy of Research and Education, Srivilliputhur, India. Email: a.s.vigneshwaran@gmail.com

Vishnuvarthanan, Department of BME, Kalasalingam Academy of Research and Education, Srivilliputhur, India. Email: gvvarthanan@gmail.com

Anitha, Department of ECE, Kalasalingam Academy of Research and Education, Srivilliputhur, India. Email: anithaa06@gmail.com

Pallikonda Rajasekaran, Department of ECE, Kalasalingam Academy of Research and Education, Srivilliputhur, India. Email: m.p.raja@klu.ac.in

Yu-dong zhang, Department of Informatics, University of Leicester, Leicester, UK. Email: yudongzhang@ieee.org

Arunprasath, Department of BME, Kalasalingam Academy of Research and Education, Srivilliputhur, India. Email: arun.aklu@gmail.com algorithms, which help in the deep analysis of the pathological portion in less computational time. Some researchers have provided the different combinational state-of-the-art methodologies to identify the pathological structures for successful oncological treatment. Pham [1] suggested the Robust Fuzzy C-Means approach and this process analyses the MR brain slices that required more processing time for effective demarcation outcomes. Ahmed et al. [2] formulated the objective function of modified FCM algorithm, which provides the better MR slices analysis in more time. Pathological detection using Modified Fuzzy C - Means and Gaussian Mixture Model was epitomized by Karim et al. [3], and it is capable of demarcating the MR brain slices. Higher processing time is required by the technique suggested by the authors. The amalgamation of Modified Fuzzy C - Means (MFCM) approach and histogram based cluster design process intended by Karan et al. [4] requires improvement of time complexity. Govindaraj et al. [5] have used Fuzzy Inference System approach for carrying out the demarcation of MR brain slices, which provides lower PSNR values and higher MSE values. Moreover, a demarcation of MR slices process used Particle Swarm Optimization and Fuzzy C Means approach by Govindaraj et al. [6]. The algorithm propounded requires improvement in segmentation accuracy. Guler et al. [7] proposed an automated demarcation of MR brain slices using SOM neural network, which is related to the processing of noise input images. SOM based Leaning Vector Quantization algorithm represented by Demirhan et al. [8] requires improvement in human interaction process. The ample path detection using SOM based extended FCM approach intended by Aghajari et al. [9] needs development in processing time. Tumor and tissue detection using SOM based FKM algorithms proposed by Vishnuvarthanan et al. [10] provides ample MR brain slices analysis. Segmentation accuracy and computational time presented by SOM based FKM algorithm needs improvement. The above-mentioned are the drawbacks that can be resolved through the novel combinational framework presented in this paper.

Section II clearly illustrates the proposed methodology. Section III exposes that the proposed methodology is better when compared with the other soft computing methodologies. Section IV discusses the completion of this process.

Published By: 


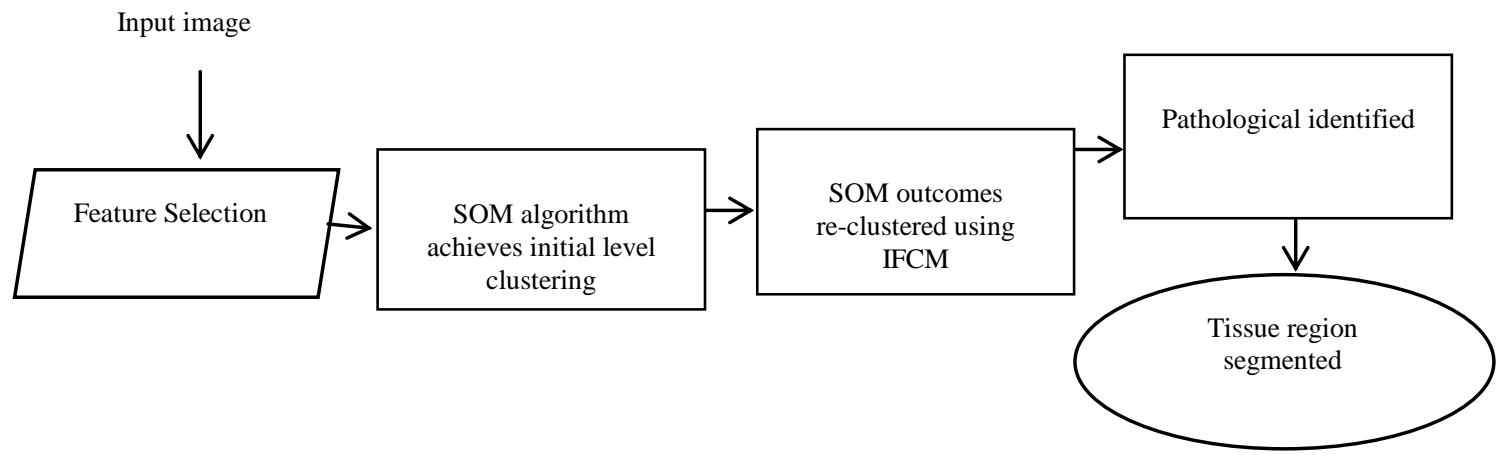

Fig. 1. Performance of the recommended SOM-IFCM methodology

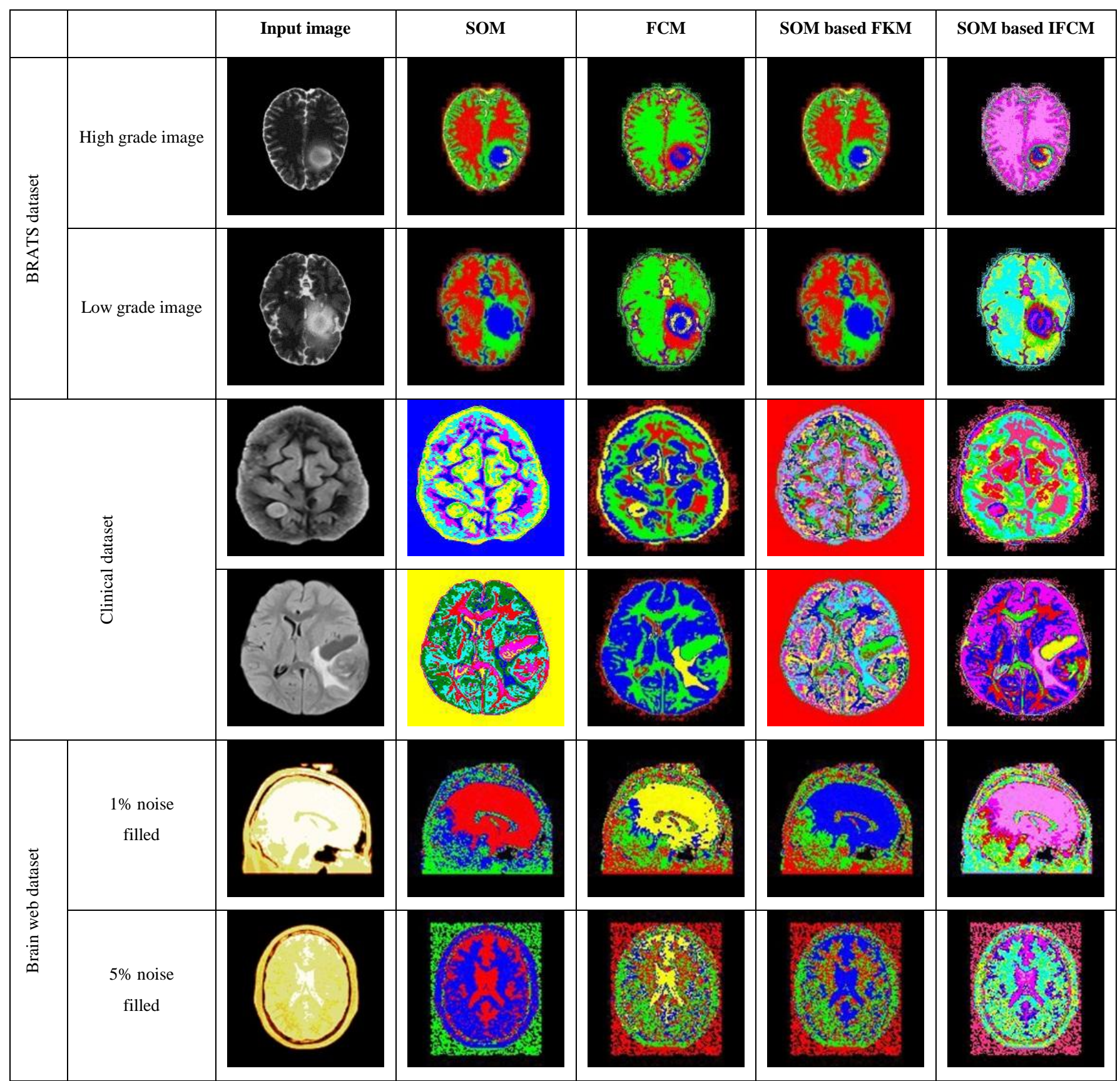

Fig. 2. Segmentation outcomes of various soft computing methodologies

\section{METHODOLOGY}

Fig 1 spotlights the combination of SOM-IFCM algorithm. SOM neural network certainly performs the classification of same set pixel and also the segmentation process. It specifies that SOM neural network provides the dimensionality reduction with the aid of the mapping function.

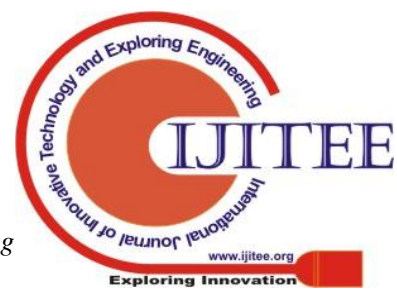


International Journal of Innovative Technology and Exploring Engineering (IJITEE) ISSN: 2278-3075, Volume-9, Issue-2S2, December 2019

Table- I: Quality metrics analysis of the proposed algorithm

\begin{tabular}{|c|c|c|c|c|c|c|}
\hline \multirow{2}{*}{ Parameters } & \multirow{2}{*}{\multicolumn{2}{|c|}{ Clinical image }} & \multicolumn{2}{|c|}{ BRATS image } & \multicolumn{2}{|c|}{ Brain web database } \\
\hline & & & High & Low & & \\
\hline MSE & 0.0767 & 0.0804 & 0.0605 & 0.0939 & 0.0593 & 0.1235 \\
\hline PSNR & 59.2809 & 59.0796 & 60.3116 & 58.4020 & 60.4010 & 57.2152 \\
\hline Jaccard Index in \% & 0.7509 & 0.8626 & 0.9169 & 0.8795 & 0.7980 & 0.8822 \\
\hline DOI values in $\%$ & 0.8577 & 0.9262 & 0.9566 & 0.9359 & 0.8877 & 0.9374 \\
\hline Computational Time in seconds & 7.5854 & 6.9007 & 6.5933 & 6.5049 & 6.7291 & 5.3836 \\
\hline Memory requirement in bytes & $2.21 \mathrm{e}+09$ & $2.20 \mathrm{e}+09$ & $2.27 \mathrm{e}+09$ & $2.19 \mathrm{e}+09$ & $2.21 \mathrm{e}+09$ & $2.19 \mathrm{e}+09$ \\
\hline
\end{tabular}

Table- II: Quality metrics analysis of the proposed algorithm

\begin{tabular}{|c|c|c|c|c|c|c|}
\hline Algorithm & MSE & $\begin{array}{c}\text { Memory } \\
\text { Requirement } \\
\text { in bytes }\end{array}$ & PSNR & $\begin{array}{c}\text { DOI } \\
\text { in bytes }\end{array}$ & $\begin{array}{c}\text { Jaccard Index } \\
\text { in bytes } \\
\text { Time seconds }\end{array}$ \\
\hline SOM & 1.98 & $2.68 \mathrm{E}+13$ & 46.3817 & 80.89 & 67.91 & 68.1 \\
\hline FCM & 0.2731 & $5.20 \mathrm{E}+12$ & 53.7832 & 81.02 & 76.69 \\
\hline SOM based FKM & 2.421 & $1.45 \mathrm{E}+14$ & 43.8567 & 86.88 & 3.7741 \\
\hline
\end{tabular}

$$
\boldsymbol{D}_{\min }(z)=\min \left\{\sum_{i}\left(x_{i}(z)-w_{i}(z)\right)^{2}\right\}
$$

Automated mapping process is utilized as $8 \times 8$ map function, which is to inspire this process with the aid of the input vector and nearest neighbour. We obtained the nearest neighbour with the help of equation (1).

$$
w_{i}(z+1)=w_{i}(z)+\alpha(z) *\left(i-w_{i}(z)\right)
$$

Then, the end-result of SOM denotes the updated weighted vectors ' $w_{i}$ ' (refer to the equation (2)), which is obtained from Best Matching Unit (BMU) of nearest neighbour and Euclidean distance. The operational parameters of equation (3) and (4) depend upon the exponential decay function of time.

$$
\begin{aligned}
& \alpha(z)=\alpha_{0} \exp \left(-\frac{t}{2 T}\right) \\
& h(z)=\exp \left(-\frac{d i s t^{2}}{2 \sigma^{2}(t)}\right)
\end{aligned}
$$

Consecutively, the ample SOM outcomes are given to the input of improved FCM unsupervised clustering algorithm. The underlying concept of improved FCM is each and every pixels connected by the minimum Euclidean distance have obtained the nearest neighbour pixel to close the cluster centroid. In clustering process, the equivalent pixels are associated with the same membership values. This proposed algorithm has the enhanced membership values that are coherence to the input vectors (for quickly convergence process). The FCM was recommended by Dunn [11] and later extended by Bezdek [12], which has now been modified and the same is given in equation (5).

$$
J(U, M)=\sum_{i=1}^{c} \sum_{j=1}^{N} U_{i j}^{m}\left\|w_{i}-c_{i}\right\|^{2}
$$

An enhanced FCM objective function is denoted as $J$, which is determined by the membership function with squared Euclidean distance of iterative optimization approach. The updated membership function of improved FCM is employed with the help of the equation (7) and (8).

$$
\begin{aligned}
& U_{i j}^{m}=\frac{\left(u_{i j}\right)^{m}}{1+\alpha d_{i j}} \\
& u_{i j}=\left[\left(d_{i j}\right)^{1 / m-1} \sum_{l=1}^{k}\left(\frac{1}{d_{i l}}\right)^{1 / m-1}\right]^{-1} \\
& c_{i}=\frac{\sum_{i=1}^{N} U_{i j}^{m} x_{i}}{\sum_{i=1}^{N} U_{i j}^{m}}
\end{aligned}
$$

Here, $c_{i}$ refers to the cluster center and also $u_{i j}$ refers to the membership function. Consequently, the efficient segmentation outcomes are delivered by the proposed algorithm, and they are obtained using the modified membership function, which delivers equivalent pixels associated with the same set.

\section{RESULT AND DISCUSSION}

The suggested SOM based IFCM methodology processes the BRATS-2013 challenge medical images [13], brain web dataset [14] and the clinical dataset. A total of 112 input medical images are operated by the single framework of novel methodology, and the proof of efficiency of this algorithm is evaluated by quality metrics like Dice overlap Index, Jaccard index (TC), Peak Signal to Nosie Ratio and Mean Squared Error [15]. Fig 2

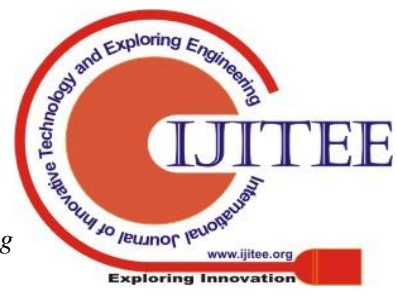


reveals the demarcation outcomes of SOM based IFCM methodology, and it is compared with SOM and FCM algorithms. Table I denotes the quality metrics of SOM based IFCM algorithm, which is useful to analyze the ample segmentation process. Table II exemplifies the performance of novel SOM based IFCM algorithm, which provides better segmentation accuracy in the form of Jaccard index when compared with the other soft computing approaches.

\section{CONCLUSION}

In this paper, a novel SOM based IFCM algorithm is offered, which needs less processing time for 112 input medical MR slices. Successively, IFCM approach is entirely enhanced by SOM outcomes. The combinational novel segmentation approach proffers better brain MR slice analysis when compared with other soft computing algorithms like SOM and FCM. SOM-IFCM approach mandates minimal processing time for identifying the pathological structures, and is mitigated in Fig 2 and Table I. Precise pathological portions in brain MR slices are represented by the SOM-IFCM algorithm, which has also delivered uppermost Jaccard index and DOI values (refers to table II), and it can sure be a matter of importance during the diagnosis of patients.

\section{ACKNOWLEDGMENT}

The authors thank the Department of Electronics and Communication Engineering of Kalasalingam Academy of Research and Education, Tamilnadu, India for permitting to use the computational facilities available in open source research laboratory. Also, the authors thank KGS Advanced MR \& CT Scan - Madurai, Tamilnadu, India, for supporting the research with the patient information.

\section{REFERENCES}

1. D.L. Pham, "Spatial models for fuzzy clustering," Computer Vision and Image Understanding, vol. 84, 2001, pp. 285-297.

2. M.N. Ahmed, A.A Farag, N. Mohamed, T. Moriarty, and S.M. Yamany, "A modified fuzzy c-mean algorithm for basis field estimation and segmentation of MRI data," IEEE Transactions on Medical Imaging, vol. 21, 2002, pp. 193-199.

3. K. Karim, and M. Mohamed, "Image Segmentation by Gaussian Mixture Models and Modified FCM Algorithm," The International Arab Journal of Information Technology, vol. 11, 2014, pp. 11-17.

4. R. Karan, S. Nitesh, S.K. Pankaj, and K. Amith Mishra, "A fully automated algorithm under modified FCM framework for improved brain MR image segmentation," Magnetic Resonance Imaging, vol. 27, 2009, pp. 994-1004

5. Govindaraj Vishnuvarthanan, Murugan Pallikonda Rajasekaran, "Segmentation of MR brain images for tumor extraction using fuzzy," Current Medical Imaging Reviews, vol. 9, 2013, pp. 2-6.

6. V. Govindaraj, and P.R. Murugan, "A complete automated algorithm for segmentation of tissues and identification of tumor region in T1, T2, and FLAIR brain images using optimization and clustering techniques," International Journal of Imaging Systems and Technology, vol. 24, 2014, pp. 313-325.

7. I. Guler, A. Demirhan, and R. Karakıs, "Interpretation of MR images using self-organizing maps and knowledge-based expert systems," Digital Signal Processing, vol. 19, 2009, pp. 668-677. DOI: https://doi.org/10.1016/j.dsp.2008.08.002.

8. A. Demirhan, M. Toru, and I. Guler, "Segmentation of tumor and edema along with healthy tissues of brain using wavelets and neural networks," IEEE Journal of Biomedical and Health Informatics, vol. 19, 2015, pp. 1451-1458. DOI: 10.1109/JBHI.2014.2360515
9. E. Aghajari, and G.D. Chandrashekhar, "Self-Organizing Map based Extended Fuzzy C-Means (SEEFC) algorithm for image segmentation," Applied Soft Computing, vol. 54, 2017, pp. 347-363. DOI: https://doi.org/10.1016/j.asoc.2017.01.003

10. G. Vishnuvarthanan, M.P. Rajasekaran, P. Subbaraj, and A. Vishnuvarthanan, "An unsupervised learning method with a clustering approach for tumor identification and tissue segmentation in magnetic resonance brain images," Applied Soft Computing, vol. 38, 2016, pp. 190-212. DOI: https://doi.org/10.1016/j.asoc.2015.09.016

11. J.C. Dunn, "A fuzzy relative of the ISODATA process and its use in detecting compact, well-separated clusters," J. Cybernetics, vol. 3, 1974, pp. 32-57.

12. J.C. Bezdek, "Pattern Recognition with Fuzzy Objective finction Algorithms," Plenum Press, New York, 1981.

13. Menze et al. "The Multimodal Brain Tumor Image Segmentation Benchmark (BRATS)," IEEE Trans. Med. Imaging, 2015.

14. D.L. Collins, A.P. Zijdenbos, V. Kollokian, J.G. Sled, N.J. Kabani, C.J. Holmes, and A.C. Evans, "Design and Construction of a Realistic Digital Brain Phantom," IEEE Transactions on Medical Imaging, vol. 17, 1998, pp. 463-468.

15. S. Vigneshwaran, Vishnuvarthanan Govindaraj, Pallikonda R. Murugan, Yudong Zhang, and Thiyagarajan Arun Prasath, "Unsupervised learning-based clustering approach for smart identification of pathologies and segmentation of tissues in brain magnetic resonance imaging," International Journal of Imaging Systems and Technology, 2019, pp. 1-18.

\section{AUTHORS PROFILE}

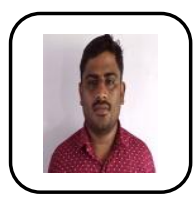

Vigneshwaran profile is a full time research scholar in the Department of Electronics and Communication Engineering at Kalasalingam University. He has completed his M.E in Applied Electronics at Anna University in 2015 and B.E in Electronics and Communication Engineering at Sudharsan Engineering College in 2012, both located at Tamilnadu, India. His primary research focus is upon medical image processing.

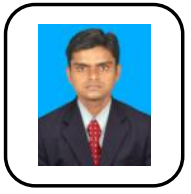

Vishnuvarthanan is an Associate Professor in the Department of Instrumentation Engineering at Kalasalingam University. Dr.Vishnuvarthanan received his Ph.D. in Electronics and Communication Engineering from Kalasalingam University, Tamilnadu in 2015 and completed his Bachelor of Engineering in Instrumentation and Control Engineering with first class in the year 2007 from Arulmigu Kalasalingam College of Engineering, Virudhunagar, Tamilnadu and Masters in VLSI with distinction in the year 2009 from Bharath University, Chennai, Tamilnadu. His areas of interest are Medical image processing and signal processing, and he has significant publications pertaining to these domains.

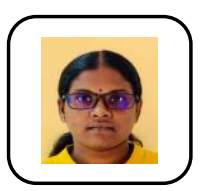

Anitha has been awarded her $\mathrm{PhD}$ degree from Kalasalingam Academy of Research and Education (Kalasalingam University) in 2019 for her research work in the area of medical image processing. She received her Master's degree in Digital Communication and Networking in 2015 and Bachelor's degree in Electronics and Communication Engineering in 2006. She has three years of work experience as software programmer and two years of teaching experience, also an expertise in students' counselling. She is an active IEEE Member of Pothigai Subsection and IEEE Solid-State Circuits Society Member of IEEE Madras section from March 2018.

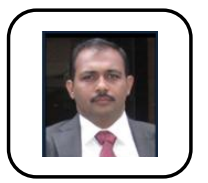

Pallikonda Rajasekaran is a Professor in Department of Electronics and Communication Engineering at Kalasalingam University. Dr. Pallikonda Rajasekaran received his Ph.D. in Electrical and Electronics Engineering from Anna University, Chennai in 2009, his M.Tech. in Biomedical Signal Processing and Instrumentation from SASTRA University in 2002 and his B.E. in Electronics and Instrumentation Engineering from Bharathidasan University (Shanmuga College of Engineering) in 2001. Dr. Pallikonda Rajasekaran research interests include biomedical instrumentation, image processing, wireless sensor networks, high performance computing, sensor systems, cloud computing, semantic networks. $\mathrm{He}$ has published

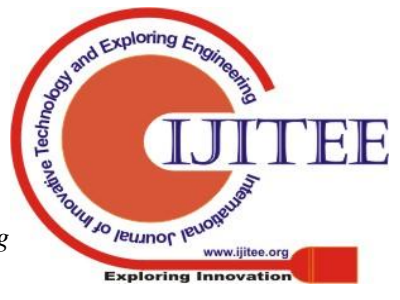


numerous technical papers in refereed conferences and journals in these areas

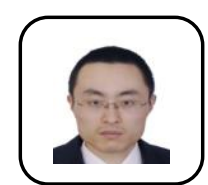

Yu-Dong Zhang received his $\mathrm{PhD}$ degree from Southeast University in 2010. He worked as a Postdoc from 2010 to 2012 in Columbia University, USA, and as an assistant research scientist from 2012 to 2013 at Research Foundation of Mental Hygiene (RFMH), USA. He served as a full professor from 2013 to 2017 in Nanjing Normal University, where he was the director and founder of Advanced Medical Image Processing Group in NJNU. Now he serves as Professor in Department of Informatics, University of Leicester, UK. He was included in "Most Cited Chinese researchers (Computer Science)" by Elsevier from 2014 to 2018 . He was the 2019 recipient of "Highly Cited Researcher" by Web of Science. He won "Emerald Citation of Excellence 2017" and "MDPI Top 10 Most Cited Papers 2015". He was included in "Top Scientist" in Guide2Research. He published over 160 papers, including 16 "ESI Highly Cited Papers", and 2 "ESI Hot Papers". His citation reached 10096 in Google Scholar, and 5362 in Web of Science. He is the fellow of IET (FIET), and the senior members of IEEE and ACM. He is the editor of Scientific Reports, IEEE Transactions on Circuits and Systems for Video Technology, etc. He served as the (leading) guest editor of Information Fusion, Neural Networks, IEEE Transactions on Intelligent Transportation Systems, etc. He has conducted many successful industrial projects and academic grants from NSFC, NIH, Royal Society, and British Council.

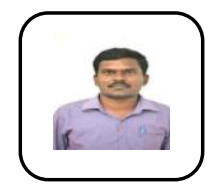

Arunprasath profile is an Associate Professor in the Department of Electrical and Electronics Engineering at Kalasalingam University. Dr. Arunprasath received his Ph.D. in Electronics and Communication Engineering from Kalasalingam University, Tamilnadu in 2015 and completed his Bachelor of Engineering in Electrical and Electronics Engineering with first class in the year 2006 from Anna University, Tamilnadu and Masters in Applied Electronics with distinction in the year 2009 from Anna University, Tamilnadu. His area of interest is Medical image processing, and he has appreciable publications in this area. 